\title{
La iniciativa BRICS y China: entre la emergencia y la irrelevancia
}

The BRICS initiative and China: between emergence and irrelevance A iniciativa BRICS e a China: entre a emergência e a irrelevância

\author{
Javier Vadell (1) \\ (1) Pontifícia Universidade Católica de Minas Gerais
}

\begin{abstract}
The objective of this article is to analyse the BRICS phenomenon from a critical perspective. The leading problem of the article is to explore how the mainstream IR and IPE interpret the emergence of BRICS as an "economic and political formation" of the international system. The main questions are: do the BRICS challenge the liberal Western order? And, how does this change affect the development and global insertion of periphery countries? W estate that the process of BRICS' institutional strengthening and its potential to expand as BRICS plus is not an isolated process. Hence, it cannot be understood without taking into consideration China's leading role in the establishment new global institutions, which implies changes in global hegemony.
\end{abstract}

\section{Keywords}

BRICS, emerging powers, China, international relations, hegemony.

JEL Codes F50, F55, F60.

\section{Resumo}

O objetivo deste artigo é analisar os BRICS a partir de uma perspectiva crítica. O trabalho começa apresentando o surgimento dos BRICS como uma "formação econômica e política", segundo as teorias tradicionais das RIs e da EPI. As questões principais são: os BRICS desafiam a ordem liberal Ocidental? De que maneira essa transformação afeta o desenvolvimento e a inserção global dos países periféricos? A nossa hipótese é que o processo de fortalecimento institucional de los BRICS e a possibilidade de expansão para um BRICS plus não é um acontecimento isolado. Isto é, não se pode interpretar os BRICS sem levar em consideração o papel de liderança da China no estabelicimento de novas instituições globais, que implicam em mudanças na hegemonía global.

\section{Palavras-chave}

BRICS, potências emergentes, China, relações internacionais, hegemonia.

Códigos JEL F50, F55, F60.

\section{Resumen}

El objetivo de este artículo es analizar los BRICS a partir de una perspectiva crítica. El trabajo comienza presentando el surgimiento de los BRICS como una "formación económica e política" según las teorías tradicionales de las RRII y de la EPI . Las preguntas principales son: ¿en qué medida los BRICS desafian el orden liberal Occidental? ¿de qué manera este cambio afecta el desarrollo y la inserción global de los países periféricos? Nuestra hipótesis es que el proceso de fortalecimento institucional de los BRICS y la possibilidad de expansión hacia un BRICS plus no es un acontecimiento aislado. Es decir, no se puede interpretar los BRICS sin considerar el rol de liderazgo de China en el establecimiento de nuevas instituiciones globales, que implican en cambios en la hegemonía global.

\section{Palabras Clave}

BRICS; Potencias Emergentes; China; Relaciones Internacionales; Hegemonía.

Códigos JEL F50, F55, F60. 


\section{Introducción}

En épocas de transformaciones sociales y económicas las herramientas teórico/conceptuales que se utilizaban para comprender los fenómenos sociales son desafiadas. Nuevas configuraciones, nuevos escenarios y cambios sorpresivos colocan en cuestión los aparatos epistemológicos, teóricos y metodológicos existentes. El interregno, parafraseando a Antonio Gramsci, como intervalo de incerteza, se presenta como una oportunidad de curiosa flexibilidad cognitiva para interpretar fenómenos nuevos.

En el área de Relaciones Internacionales (RRII) y de la Economía Política Internacional (EPI) este proceso no es diferente. La moderna área de EPI nace en la década de 1970 (Cohen, 2014) en un contexto de crisis económica y de gobernanza del capitalismo global que cuestionaban la primacía económica de los Estados Unidos de América (EEUU) frente al desafío de la emergencia económica de sus aliados europeos y de Japón. El sistema interestatal reconocido como bipolar se reconfigura con el acercamiento de la República Popular de China (RPC) a los EEUU en el esfuerzo de aislar a la Unión de Repúblicas Socialistas Soviéticas (URSS).

En este sentido, podemos observar una tácita división del trabajo en el mainstream de los estudios internacionales. Mientras las RRII tenían como foco de estudio las relaciones interestatales intentando comprender el sistema bipolar y las configuraciones históricas del equilibrio de poder, la EPI pretendía, desde el trabajo seminal de Susan Strange (1970), aproximar la economía internacional a la política internacional para entender las transformaciones en el escenario internacional en la década de 1970. No obstante, esta preocupación de Strange no fue ajena al pensamiento latinoamericano de posguerra. A partir de la década de 50, el trabajo de Raúl Prebisch, Celso Furtado, Osvaldo Sunkel y otros prominentes intelectuales desde la CEPAL (Bielschowsky, 2000) y luego la(s) teoría(s) de la dependencia nunca consiguen ser incorporadas al debate del mainstream del pensamiento de las IR y de la IPE. El método histórico estructural y el presupuesto ontológico de un sistema internacional jerárquico basado en la dicotomía centro/periferia contrastan con los presupuestos del maisntream: orden anárquica, agentes racionales, evolucionismo liberal en su versión económica (Rostow, 1960) y en su versión política (Huntington, 1968) que se populariza como receta a seguir para los países periféricos con la Comisión Trilateral (Gill, 1990) y posteriormente con el Consenso de Washington (Williamson, 1990). 
Partiendo de estas premisas, el objetivo de este artículo es analizar el fenómeno BRICS en un contexto internacional conturbado económicamente y políticamente. Este trabajo es una invitación a abrir nuevas interpretaciones en un período de transición, marcados por movimientos de placas tectónicas de las estructuras sociales desiguales e inestables en la trayectoria de desarrollo de los países periféricos, en un período de fin de del boom de las commodities (Vivares, 2018).

La pregunta principal del artículo es, en primer lugar, distinguir y analizar cómo las RRII y la EPI del mainstream interpretan la emergencia de los BRICS como "formación económica y política" (Nayyar, 2016) del sistema internacional. En otros términos, pretendemos cuestionar el debate contemporáneo de las principales corrientes de pensamiento del mainstream de los estudios internacionales que giran en torno de la pregunta: ¿en qué medida los BRICS desafían el orden liberal económico Occidental (Liberal Western Order) y como este cambio afecta el desarrollo e inserción global de los países periféricos? En segundo lugar, a partir de este cuestionamiento, nuestra segunda pregunta es: ¿se puede hablar de un fortalecimiento o un debilitamiento de la formación política y económica BRICS?

Estas cuestiones nos remiten, en primer lugar, a la discusión teórica de la "emergencia" en las relaciones internacionales y, en segundo lugar, a analizar el grado de relevancia de los BRICS en el proceso de 'emergencia'. En este sentido, el fenómeno BRICS es importante en la medida en que su evolución institucional (Vadell e Ramos, 2019), fundamentada en el colective financial statecraft (Roberts et al., 2018), respalda un conjunto de demandas por reformas institucionales del orden liberal económico Occidental y, al mismo tiempo, promueve, bajo el liderazgo de la República Popular de China (RPC), un nuevo orden normativo basado en principios reguladores y de Cooperación Sur-Sur (CSS) que desafían los fundamentos neoliberales.

Nuestra hipótesis es que este proceso de fortalecimiento institucional de los BRICS y su potencial ampliación para un BRICS-plus (BRICS, 2017), no es un proceso aislado y no puede ser estudiado sin llevar en consideración el protagonismo de China en la construcción de un conjunto de instituciones minilaterales (Wang, 2014) y una multilateral, el Asian Infraestructure, Investment Bank (AIIB), que paradojalmente subsume a la formación política BRICS en una miríada de instituciones paralelas creadas y estimuladas por China. 
Para este objetivo, este capítulo se divide en tres partes centrales, la introducción y las conclusiones. La segunda sección, enfoca en las consideraciones teóricas sobre el fenómeno de la emergencia, desde las interpretaciones del mainstream hasta llegar a la teoría crítica de la IPE con el objetivo de analizar el fenómeno de los BRICS. La tercera parte presenta la evolución del fenómeno BRICS, su emergencia y evolución y los desafíos futuros en un cuadro institucional mayor liderado por China.

\section{Consideraciones teóricas}

\subsection{La cuestión de la emergencia para las teóricos de RRII y EPI del mainstream}

En el siglo XXI, el fenómeno de la ascensión de las "potencias emergentes" y los "mercados emergentes" ha cuestionado la distribución de poder existente y estimulado un debate sobre cuál es el tipo de emergencia o "re-emergencia" que se observa en el sistema internacional contemporáneo y cuáles serán las consecuencias de estas transformaciones. Este debate se presentó como una desafío teórico y metodológico para las corrientes tradicionales de las RRII y la IPE. En este sentido, es crucial observar y analizar cómo las teorías del mainstream de las RRII y de la IPE interpretam la evolución de los BRICS, su institucionalización y el rol de los estados emergentes'.

La problemática de la emergencia tiene su lugar en las teorías de las corrientes teóricas tradicionales. Para los pensadores del realismo, que ven el sistema internacional a partir de un contexto anárquico y a las relaciones internacionales como un juego de suma cero donde prevalece la importancia de las ganancias relativas entre los actores, se espera la posibilidad de un conflicto entre los países emergentes y la potencia hegemónica (Mearsheimer, 2001; Mearsheimer, 2006; 2010; Layne, 2018). Para esta vertiente, la ascensión de las potencias emergentes, y especialmente de China, implicará en cambios en la distribución de poder con un desenlace de conflicto muy probable, para los más radicales, inevitable. En esta dirección, Graham Allison moderando un poco el determinismo, alerta sobre la trampa de Tucídides (Thucydides Trap) (Allison, 2017). El punto de partida es la Grecia antigua donde el ascenso de Atenas y el temor que esto inculcó en 
Esparta hizo inevitable la guerra. Según el autor, en los últimos 500 años hubo 16 casos de potencias emergentes intentando ganar su espacio y colocando un desafío a la potencia dominante. El resultado es que, en 12 de esos casos hubo conflicto bélico. La inminencia del conflicto es temperada con la posibilidad de una brecha de acción de los actores estatales frente a los constreñimientos del sistema.

En esta dirección, al espectro de la ascensión de China en los últimos años se le suma un intenso debate sobre la declinación de los EEUU. Christopher Layne (Layne, 2018), reafirma los postulados realistas más rígidos en relación a los peligros que enfrenta la Pax Americana. Para Layne "El destino del orden internacional está estrechamente vinculado a las dinámicas de transición de poder." (Layne, 2018). Presuponiendo que el orden normativo internacional liberal esté intrinsecamente relacionado a la Pax Americana, si este orden normativo se desgasta, la decadencia de los EEUU ocurrirá necesariamente. Para Layne, habiendo profundos cambios en el status geopolítico de la hegemonía estadounidense, con el surgimiento de las potencias emergentes y de China especialmente, no hay posibilidades de preservar el status quo de la Pax Americana y su orden internacional "liberal".

Sin embargo, el realismo se nutre de otra vertiente influyente en la teoria de las RRII y de la EPI que reconoce la jerarquía entre los Estados e inclusive afirma que una potencia dominante es necesaria para mantener el sistema politico y económico internacional estable ${ }^{1}$. No es la balanza de poder y si la preponderancia del poder de un líder lo que promueve la estabilidad. Como Organsky destaca: "podemos entender ahora por qué la paz mundial coincide com períodos de supremacía indiscutible de poder, mientras que los períodos de balanza de poder han sido de guerra" (1968).

Según este abordaje, el período de transición de una potencia hegemónica a otra está marcado historicamente por conflicto y guerra, salvo raras excepciones. Como Organski (1968) destaca, la transferencia pacífica de poder/liderazgo de Gran Bretaña a los EEUU fue una excepcionalidad. Este argumento de Organski se aproxima a la hipótesis de la trampa de Tucídides de Allison (2017). Kim and Gates destacan que la teoría de las transcisiones hegemónicas se basa en dos puntos. El primero es que el poder de un país proviene de su desarrollo interno, y como estas naciones se desarrollan de manera desigual existe diferencia en el poder relativo entre ellas. El segundo

1 En relación a la estabilidade del sistema económico, más adelante abordaremos la teoría de la estabilidad hegemónica. 
punto es que el sistema internacional es definido por la nación dominante, el hegemon. "Aquellas ocasiones en que un poder ascendente supera al poder dominante se denominan transiciones de poder" (Kim e Gates, 2015).

Para la teoría de la transição hegemónica existen determinantes externos y domésticos esenciales para ser una nación que aspire a ser hegemón. Ejemplo de ello son el "carácter nacional", la población, el territorio y el desarrollo económico y tecnológico de un país. En este sentido, es la naturaleza de la estabilidad sistémica el punto de contacto con la Teoría de la Estabilidad Hegemónica (TEH). De acuerdo con Organiski (1968), la inestabilidad ocurre cuando la dinámica del hegemón es desafiada. Para los teóricos de la TEH, la inestabilidad ocurre cuando el hegemón no asume la resposabilidad de ofrecer los bienes públicos internacionales, sea por negligencia o incapacidad material de hacerlo (Kindleberger, 1973; 1981; Gilpin, 1987; 2001).

El orden económico liberal no puede ser autorregulado, porque su resultado sería el caos o la entropia, parafraseando a Schweller (Schweller, 2014). Así como en las esferas domésticas nacionales existen instituciones para reglamentar la moneda, las transacciones, la seguridad, etc., en el plano global la presencia de un líder hegemónico es imperiosa y necesaria. Con el tiempo, y en consonancia con los cambios de la pós-guerra fría, autores como Gilpin reconocían la posibilidad de una administración que incluyese más actores, como puede serlo el G7 (Gilpin, 2000; 2001).

Por otro lado, la perspectiva liberal institucionalista, si bien parte del supuesto de un sistema internacional anárquico, destaca la institucionalidad normativa y la interdependencia de los actores estatales en este marco institucional compartido de valores liberales. Robert Keohane, su representante liberal, fue el creador de la expresión TEH en la década de 1980. Frente a una declinación hegemónica, Keohane deposita su confianza en una fuerte y consolidada institucionalidad liberal para administrar la economía internacional (Keohane, 1984). No obstante, después del fin de la URSS, en el "momento unipolar" (Krauthammer, 1990), el mainstream de las RRII y de la EPI tuvieron que enfrentar el desafío intelectual de los cambios políticos y económicos producidos en el sistema capitalista global: la crisis del modelo neoliberal en 2008 y la ascensión de los países emergentes. Para estos académicos liberales institucionalistas, las potencias emergentes tienen toda la posibilidad de 'ganar espacios' dentro del orden internacional liberal. 
Heredero del legado de Keohane (1984), John Ikenberry (2018) sostiene que las potencias emergentes han ascendido dentro de las normas liberales internacionales y ello los compromete a respetarlas. Autores de esta línea destacan la posibilidad que los EEUU realicen una estrategia de lock-in. Es decir, si las instituciones de la Pax Americana son reformadas, a China y a otras potencias emergentes no Occidentales les resultará más atractivo permanecer en el orden internacional creado en el post 1945 que derrumbarlo (Brooks et al., 2012/13).

\subsection{Potencias medias y potencias emergentes: ¿estabilizadores versus desestabilizadores?}

Fue a partir de mediados del siglo XX que comenzaron a surgir los primeros debates teóricos sobre las potencias medias y emergentes desde la academia canadiense (Cooper e Higgott, 1990; Cooper et al., 1993; Huelsz, 2009), que se profundizan a fines de década de 1990 y, principalmente, en el siglo XXI. Un punto en común en el debate del nuevo milenio es la diferenciación entre las potencias medias tradicionales y las "emergentes" (Nolte, 2007; Huelsz, 2009). Estas últimas tienen la característica de ser grandes Estados, con mucho territorio y muy poblados que, si bien son considerados "en desarrollo", sorprenden por su crecimiento económico y su potencial. Es el caso de los BRICS.

En un estudio sobre el debate de los BRICS y las instituciones multilaterales, Gregory Chin identifica tres fases. La primera parte de la cuestión de "cómo el grupo de países emergentes contemporáneos asumió los acuerdos multilaterales establecidos y el orden global (Chin, 2015). Podemos colocar como ejemplo los temas de la diplomacia de nicho de las potencias medias tradicionales (Gilley e O'neil, 2014), el rol de las potencias medias en las instituciones de gobernanza Occidental y sus políticas exteriores (Cooper, 1997) y el concepto de "middlepowermanship" (Cox, 1989; Hurrell, 2000; Alexandroff e Cooper, 2010; Cooper, 2013).

Un segundo foco destaca el aspecto comportamental de los países emergentes para saber en qué medida estos Estados se incorporaban, o no, a las instituciones multilaterales. Es decir, "Si las potencias emergentes se comportan como potencias defensoras del status quo o como revisionistas" (Chin, 2015). La tercera fase se aproxima más al objetivo de este trabajo 
que trata del rol de los BRICS frente a las instituciones existentes. $\bigcirc$ sea: "si las potencias emergentes están intentando reformular algunas normas y reglas globales, incluso mientras internalizan selectivamente algunas de las reglas globales establecidas" (Chin, 2015).

Eduard Jordaan se pregunta si las potencias emergentes están promoviendo acuerdos multilaterales alternativos. Su contribution critica es el concepto de "potencia media emergente", incorporando anteriores discusiones sobre las potencias medias y las actuales expectativas en torno a la ascensión de China. (Jordaan, 2017),

En el cuestionamiento del concepto de potencia media, y su utilidad en el mundo contemporáneo, Jordaan destaca la clasificación de las "potencias medias" en términos de comportamiento y hegemonía. Para Jordaan, el concepto de potencia media es "demasiado escurridizo" como categoría. El autor propone el concepto de "mid-range states" para países sostenes del proyecto hegemónico liberal. Sin embargo, no hay una clara respuesta sobre cuáles factores (producto nacional bruto, poder regional, liderazgo, capacidades diplomáticas o comportamiento moral) deberían ser mensurados y cómo ponderar los diversos componentes de las capacidades estatales (Jordaan, 2017, p. 2).

En términos de hegemonía, el concepto contribuye al debate sobre las características estabilizadoras y/o desestabilizadoras de las potencias medias. De hecho, el abordaje de Jordaan enfatiza el rol de los agentes y sus comportamientos en la política internacional. En esta dirección, el autor diferencia tres tipos de potencias medias:

El primer tipo es la potencia media como un estabilizador. Es decir, como "seguidores del hegemon" (Cooper, 1997; Jordaan, 2017) con una posición pro status quo. Durante la Guerra Fría, Robert Cox destacacó la tarea de la potencia media, Japón, como sostén y apoyo del orden internacional (Jordaan, 2017, p. 5).

El segundo enfoque sobre las potencias medias se relaciona a su posición ambivalente respecto a la hegemonía de los EEUU en las instituciones de governanza del capitalismo global. La preferencia por la adhesión a las normas e instituciones existentes ha variado de un país para otro desde que terminó la Guerra Fría y, especialmente, en el nuevo milenio. Algunas potencias medias emergentes, por ejemplo, no están satisfechas con con ciertas normas y reglas del sistema de gobernanza actual, pero eso no necesariamente significa que se oponen a ls organizaciones internacionales 
que encarnan estas normas y reglas. Un buen ejemplo es el debate en torno a las reformas del FMI y el Banco Mundial que contemplen una mayor participación de los emergentes.

Finalmente, la tercera perspectiva entiende que los intereses de las potencias medias son de contener a las grandes potencias. En este enfoque, las potencias medias prefieren un orden multipolar internacional, porque esto les permitiría una mayor influencia en temas específicos (Ramos et al., 2012). El problema con este modelo estático es la imposibilidad de utilizarlo para comprender la compleja dinámica de la economía global contemporánea y la ascensión de China en la 'era Trump'. En la actualidad, donde se observa un cambio de roles de las potencias, seguir a los EEUU no significa necesariamente apoyar un 'orden internacional liberal'. El escenario internacional se tornó más complejo y contradictorio. En otros términos, se debe prestar atención a los cambios en la estrutuctura económica y en la geopolítica e incorporarlos al análisis para entender el fenómeno de la emergencia y la constitución de la hegemonía en el sistema capitalista.

\subsection{EPI crítica y la subsunción de los BRICS}

Para entender la importancia de los BRICS en el actual proceso de transformación del sistema capitalista global debemos, en primer lugar, ir más allá de los procesos de acción racional orientados por los agentes estatales, considerados en función de la cuantificación de capacidades, en segundo lugar, como orientación metodológica, los agentes deben ser entendidos a partir de un enfoque integral que contemple las transformaciones de la estructura, llevando en consideración la economía, la política, la historia, las ideas, la cultura y las ideas.

La relevancia económica de los BRICS y el ascenso económico de sus miembros, especialmente de China e India, es parte de un proceso histórico persistente de expansión global del capitalismo. Como destaca Li:

La premisa de la EPI muestra que todos los Estados y los mercados están interconectados en un sistema global de producción, intercambio y distribución, y la EPI investiga las formas en que los Estados y mercados están conectados unos con otros y las estructuras que han evolucionado para conectarlos. La ascensión de China y de otras potencias emergentes han perturbado la distribución convencional de poder y la manera en que los Estados y mercados se relacionan. (Li, 2019a) 
Por lo tanto, la teoría de Sistema Mundo y el abordaje neo-gramsciano de EPI proporcionan una interpretación histórica y holística para la comprensión del los países emergentes y las posibilidades de mobilididad ascendente o descendente en la jerarquía del sistema mundial moderno.

Cambios en la economía capitalista y en el ajedrez geopolítico de las grandes potencias pueden alterar los incentivos de los países semiperiféricos individualmente o colectivamente con el objetivo de cambiar o modificar las reglas. Sin embargo, como fue destacado, los países emergentes pueden querer cambiar algunas reglas sin modificar las organizaciones internacionales. Las potencias emergentes pueden entender que normativas internacionales no reflejan la ecuación multipolar del sistema internacional. En este sentido, como Wallerstein afirma, el rol de la semiperifería, como arena de las "potencias medias", es crucial para entender las posibilidades de cambios en la balanza de poder global. En esta dirección, Li sostiene que "la competición entre Estados poderosos y el esfuerzo de los Estados semi-periféricos con el objetivo de ampliar su status y su poder tienden a una rivalidad interestatal que normalmente toma la forma de una balanza de poder" (Li, 2019a).

Este abordaje teórico es útil para entender el rol de las potencias emergentes en la coyuntura actual, específicamente en lo que concierne al BRICS y al rol de China en el Sistema Mundial y dentro de la formación económica y política BRICS. Una coyuntura favorable puede ofrecer oportunidades a países en un escenario de rivalidad interestatal, pero también puede crear competición entre los países emergentes. De hecho, en este contexto conturbado la "promoción por invitación" es posible, como expresa Wallerstein. Ella se refiere a la movilidad ascendente que una nación periférica o semi-periférica puede realizar en condiciones específicas. "Esta movilidad ascendente es estimulada por un ambiente externo favorable creado por el hegemon existente o por un grupo de países centrales por medio de una promoción por invitación por causa de sus propios intereses geopolíticos y geoeconómicos" (Wallerstein, 2004, p. 57).

Sin embargo, el dilema no es la promoción por invitación, sino quién o quienes invitan y en qué circunstancias. Li Xing destaca el ejemplo de China de "promoción por invitación" (2017, p. 4). Con el ingreso de la RPC al sistema capitalista en las décadas de 1970 y 1980 por intermedio de la consolidación del "socialismo con características chinas" de Deng Xiaoping, la potencia asiática se benefició en un escenario geopolítico anti 
URSS. Después de la Guerra Fría, y particularmente en el siglo XXI, algunas potencias emergentes adquirieron una fuerte identidad internacional "basada en un clara visión del orden mundial y un entendimiento de la posición de esos países en el actual orden" (2017). Además, diferentemente de las potencias medias tradicionales, las potencias emergentes son también potencias regionales y ansían influenciar en algunas áres temáticas de la agenda mundial.

Por otro lado, el aporte de Robert Cox (1981) es importante para comprender organicamente los nexos entre hegemonía, fuerzas sociales y cambio histórico. Cox reinterpreta el concepto de hegemonía de Gramsci y lo aplica el ámbito internacional, es decir, de qué manera la hegemonía doméstica dirigida por una clase, o segmento de una clase dominante, como fuerza social hegemónica, se proyecta y se expande geograficamente en escala global. Esta expansion es al mismo tiempo geográfica y transterritorial (Scholte, 2000) y comprende la relación triádica entre las ideas, las capacidades materiales y las instituciones, como "amalgama" de las dos primeras (Bieler e Morton, 2003; Li, 2019b).

El surgimiento de los BRICS en paralelo con el ascenso de China revela los vínculos estructurales y dialécticos entre las capacidades materiales, las ideas (concepciones de orden mundial) y las instituciones, es decir, el proceso triádico 'coxiano' de la reproducción hegemónica. En esta dinámica, los cambios pueden provenir de cualquiera de estas esferas e impactar en las otras. En el ejemplo de la creación y evolución institucional de los BRICS, se observa esta compleja transformación que será desarrollada en la próxima sección.

\section{BRICS: orígenes y propósito}

Desde el nuevo milenio se reforzó una dinámica de transformaciones en el sistema internacional: un gradual proceso de reordenamiento global que desafía no sólo las teorías tradicionales de RRII, sino también a la EPI crítica. En este proceso de reordenamiento, el grupo BRICS (Brasil, Rusia, India, China y Sudáfrica) surge como un importante actor en la escena política internacional.

Haciendo una retrospectiva de la conformación y evolución del grupo BRICS, se debe tener presente que las potencias emergentes no participa- 
ron activamente en las instituciones de gobernanza global hasta la creación del G-20, primero como reunión ministerial, después de la crisis de finales de la década de 1990, y sólo en el 2008 como cumbre de jefes de Estado y gobierno (Vadell e Ramos, 2019). A inicios del siglo XXI, Brasil, India, China, Sudáfrica y México gradualmente fueron invitados a la cumbre del G8 como observadores (G8+5). Sin embrago, no formaban parte de los debates de la dirección de la economía mundial (Ramos et al., 2012). En 2003 fue creado el IBAS (India, Brasil y Sudáfrica) (Giaccaglia, 2013) y en el 2006 se llevó a cabo la primera reunión entre los Ministros de Relaciones Exteriores de Brasil, Rusia, India y China. Esta concatenización de eventos y demandas por reformas en el orden internacional fertilizó el terreno para la realización de la primera cumbre de los BRICS en junio de 2009, en Ekaterinburgo.

Pasaron diez años y una pregunta natural surge: ¿Cuál es la importancia de los BRICS para el orden mundial? ¿Los BRICS están en condiciones de proponer un nuevo paradigma de desarrollo? (Costa Vazquez, 2018). Comenzando por la segunda cuestión, no se puede afirmar que el grupo BRICS propone un nuevo paradigma de desarrollo, sino que su surgimiento y consolidación institucional, via Nuevo Banco de Desarrollo (NBD), está cada vez más estrechamente relacionado a las transformaciones en el sistema económico y político global y al creciente poderío económico de China.

El punto de partida de este artículo difiere de algunos estudios que identifican el nacimiento de los BRICS a un tipo de "aversion" al sistema unipolar, que se consolida en la pós-guerra fría. Los BRICS no surgieron en la era multipolar "después del colapso de la URSS a inicios de los años '90" (Roberts et al., 2018), sino en un sistema multipolar, después de la crisis financiera de 2008. De esta manera, las "aversiones en común" no pueden caracterizarse como el "focal point" (Roberts et al., 2018) de la colaboración o cooperación entre los BRICS. Este trabajo parte de otro supuesto, que se aproxima más del argumento de un reclamo común (common claim) en una era multipolar. El diagnostico de un sistema multipolar y de la defensa de un sistema multilateral más equilibrado se encuentra en inúmeros documentos oficiales de China para el Sur Global (China, 2008; 2011; ChinaScprc, 2011; China, 2015).

En este sentido, el artículo parte de tres variables que estimularon la formación de propósitos comunes para la creación de los BRICS, que se pueden categorizar como: 
a) La crisis del paradigma neoliberal: que implica en una crisis de la globalización neoliberal y el consecuente cuestionamiento del rol de la clase financiera transnacional, especialmente después de la de 2008 (Jessop e Overbeek, 2019). En el plano politico/institucional, los países en desarrollo y los emergentes cuestionaron la administración de las crisis nacionales vía Banco Mundial (BM) y Fondo Monetario Internacional (FMI), por medio de la imposición de recetas predefinidas como el Consenso de Washington. En la cumbre de Seul del G-20, en 2010, se llegó a un acuerdo denominado "Consenso de Seul" basado en la importancia del crecimiento económico inclusivo y sustentado para la reducción de la pobreza y la ausencia de una única formula para el desarrollo (Ramos et al., 2012). En esa cúpula también se consensuaron los cambios en las cuotas y votos en el FMI.

b) Reclamos mutuos por mayor participación: por parte de los miembros de los BRICS surgieron crecientes demanda por una mayor participación en las instituciones de governanza económica global: el BM y el FMI. Como destacaron Christensen y Li: "Somos testigos de movimientos hacia nuevos patrones de EPI en términos de nuevas formaciones de alianzas resultantes de las respuestas globales a las nuevas situaciones" (Christensen and Li, 2016), también como "nuevo tipo de relaciones hegemónicas entre las potencias existentes y las emergentes" (Li 2016). BRICS, el "Segundo Mundo", Belt and Road Initiative (BRI), (Ramos et al., 2012), la Nuva Ruta de la Seda, El Nuevo Banco de Desarrollo (NDB), El Fondo de la Ruta de la Seda, y el Banco Asiático de Inversiones en Infraestructura (BAII) son ahora importantes instituciones de la gobernanza de las relaciones internacionales y la economía política internacional "simbolizando el fenómeno creciente de un orden mundial en transformación en el cual el sistema no es más dirigido y gobernado por los tratados de pós-guerra liderados por los EEUU" (BRI, 2015).

c) Rol crucial y creciente de China en la governanza global, de 'rule shaper' hacia "rule maker" (Wang, 2018; Li, 2019a): Este proceso supone un nuevo tipo de liderazgo en los asuntos de EPI, en la governança global, en las relaciones inter-estatales y entre las fuerzas sociales. La ascesión de la RPC y la nueva administración Xi Jinping, desde 2013, promovió una serie de cambios en la política externa que algunos au- 
tores entienden como un cambio substancial, con un propósito más asertivo y expansivo (Yan, 2014). Este cambio tuvo su correlato en la creación de instituciones minilaterales financieras (Wang, 2014) y una multilateral, el BAII, la consolidación del comercio internacional y un mayor estímulo a las inversiones externas. Este proceso de fortalecimiento y expansion china fue configurando lo que denominamos como: fusión y subsunción de las instituciones del BRICS a la instituciones lideradas por China.

Partiendo de estas tres variables, esta sección presentará una restrospectiva de la evolución de los BRICS, desde su creación como "formación económico y política" (Wang, 2018) hasta la institucionalización del NDB y la propuesta china de un BRICS plus. Paralelamente a la narrativa se realizará um análisis basado en la IPE crítica.

Desde la primera cumbre en 2009 el principal objetivo de los BRICS ha sido cooperar en el marco de las cumbres del G20 y resaltar la importancia de consensuar una reforma de las instituciones financieras internacionales para permitir una mayor participación de las potencias emergentes en el orden internacional (BRICS, 2009). En la segunda cumbre, los miembro del BRICS destacaron la crisis de legitimidad y la necesidad de reformas en las instituciones internacionales, particularmente de las Naciones Unidas (NNUU) y del FMI (Vadell e Ramos, 2019).

La tercera cumbre de los BRICS se llevó a cabo en Sanya en 2011. En esta reunión dos acontecimientos se destacan: a) la inclusión de Sudáfrica y b) el hecho que en ese año todos los países BRICS formaban parte del Consejo de Seguridad de las Naciones Unidas (CSNU), hecho que derivó en preocupaciones que enfocaron cuestiones de seguridad internacional, por ejemplo, sobre las consecuencias de la primavera árabe. Por primera vez en la declaración final hubo la mención explícita para promover una reforma de las NNUU (BRICS, 2010). Además, se reafirmó la importancia del G20 para la arquitectura financiera global y la necesidad de completar la Ronda Doha (BRICS, 2011, \$8).

La cuarta cumbre de los BRICS ocurrió en Nueva Delhi, en el 2012 y se caracterizó por una posición conjunta y más asertiva, continuando con los reclamos por reformas en las instituciones de governanza. Por primera vez, se discutió la posibilidad de la creación de un nuevo banco de desarrollo de los BRICS, que resultó en un compromiso conjunto para evaluar la posibilidad. (BRICS, 2011). 
La evolución en cantidad de miembros - de 4 para 5 - se observa en la quinta cumbre de los BRICS en Durban, en 2013. Este encuentro fue un punto de referencia para la institución debido a su giro geopolítico. La incorporación de Sudáfrica como interlocutor de África, abriendo las puertas para la cooperación para el desarrollo, es la gran novedad de la primera gran transformación del grupo ${ }^{2}$. Como en cumbres previas, los BRICS reafirmaron su compromiso con el multilateralismo y con la defensa de una gobernanza global más democrática por medio de reformas en las instituciones financieras internacionales, especialmente el reclamo por la reforma acordada en 2010 de las cuotas y votos en el FMI. (BRICS, 2012). Conjuntamente, los BRICS destacaron su compromiso para concluir la Ronda Doha de la OMC, como también mayor participación en las NNUU de Brasil, India y Sudáfrica. Además, expresaron el apoyo al candidato de Brasil para asumir el cargo de Director general de la OMC, Roberto Azevedo.

Estas tres demandas demuestran la voluntad por mayor participación en las instituciones de governanza econômica lideradas por las potencias occidentales. No obstante, los BRICS anunciaron la creación del Nuevo Banco de Desarrollo (NBD), cuyo objetivo es financiar "recursos para proyectos en infraestructura y en desarrollo sustentables para sus miembros y otras economías emergentes y naciones en desarrollo, complementando los esfuerzos existentes de las instituciones financieras multilaterales y las asociaciones regionales para el desarrollo y el crecimiento global" (BRICS, 2013, \$13). Paralelamente, se acordó crear el Acuerdo de Reservas de Contingencia (ARC) con U\$S 100.000 millones, cuya finalidad sería el evitar presiones de liquidez en el corto plazo y promover la cooperación entre los miembros (BRICS, 2013, §9).

En la sexta cumbre de los BRICS, realizada en Fortaleza en 2014, se inauguró una nueva era de encuentros donde las propuestas superaron a las demandas. El tema principal fue: "Inclusive Growth: Sustainable Solutions". El acuerdo derivó en la firma del establecimiento del NBD, cuyo propósito es la movilización de recursos para proyectos de infraestructura y desarrollo sostenible en los países miembros, para economías emergentes y otros en desarrollo (BRICS, 2013, \$10). El NBD autorizó un capital inicial de U\$S 100.000 millones con un capital inicial suscrito de U\$S 50.000 millones, "divididos igualmente entre los miembros fundadores" (BRICS, 2014, \$11).

2 El tema central fue: "BRICS and Africa: Partnership for Development, Integration and Industrialisation". 
Adicionalmente, los participantes firmaron el BRICS-ARC (con un capital de U\$S 100.000 millones), un Memorandum de Entendimiento para la Cooperación Técnica entre Agencias de Crédito y varios acuerdos de garantías para las exportaciones.

En la cumbre de Ufa, en 2015, hubo muchas expectativas en relación a la promoción del comercio intra-BRICS y en la cooperación financiera y de inversiones por intermedio de dos proyectos: a) la creación de Agencias de créditos para la exportación de los BRICS y; b) un rol creciente del "BRICS Interbank implementation of the BRICS Framework for Trade and Investment Cooperation". Se observa también el contacto más claro entre el NBD-ARC y la iniciativa financiera multilateral liderada por China: el BAII ${ }^{3}$, institución fundamental para el financiamiento de los proyectos en infraestructura de la iniciativa de la Franja y Ruta (IFR) ${ }^{4}$ (BRICS, 2014, \$13 and \$14). Los participantes debatieron detalles y posibilidades de colaboración entre estas nuevas instituciones (BRI, 2015).

Las demandas por un orden más justo y que contemple mayor participación de los BRICS en las instituciones internacionales se reiteran en la cumbre de Goa, destacando el la importancia de la cooperación en un "orden internacional multipolar" (BRICS, 2016).

Conjuntamente al diagnostico de un sistema multipolar y a las demandas por un multilateralismo más democrático en las relaciones internacionales, los temas sobre seguridad internacional se hicieron más presentes en las declaraciones: el conflicto en Siria, la necesidad de una solución de dos Estados para el conflicto Israel-Palestina, los desafíos de promover la paz en Afganistán y el combate al tráfico de drogas.

Sin embargo, el proceso de fortalecimiento institucional pasa por las cuestiones financieras, especificamente en torno del NBD. Se destacan en esa área: a) una propuesta de crear una agencia de rating de los BRICS (BRICS, 2015, \$15), juntamente con la creación de una plataforma de discusión de Agencias de créditos a las exportaciones (BRICS, 2016, \$44), y b) el establecimiento de un Comité de Cooperción Aduanera de los BRICS en el marco de la Strategy for Economic Partnership.

3 El BAII fue creado en el mismo año que el NBD. Los miembros fundadors fueron 57 países (algunos de ellos aliados históricos de los EEUU como Gran Bretaña, Alemania y Francia) y es claramente liderado por China, que posee poder de veto. La sede se encuentra en Beijing (https://www.aiib.org/en/news-events/news/2016/20160625_003.html).

4 Yi dai yi lu ou One Belt One Road en inglês que pasó a utilizar la expresión Belt \& Road Initiative com a sigla BRI. 
La novena cumbre de los BRICS fue realizada en Xiamen, China. En ella se firmaron importantes documentos para fortalecer la institucionalización financiera. a) un plan de acción para la innovación y cooperación (20172020); b) un marco estratégico para la cooperación aduanera entre los BRICS; y c) un Memorandum de Entendimiento de Cooperación (MOU) entre el Consejo de Negocios de los BRICS y el NBD. Hubo acuerdos también en torno de cuestiones monetarias como el Local Currency Bond Markets de los BRICS y el establecimiento futuro de un Fondo de Obligaciones en moneda local de los BRICS (BRICS, 2016, \$13), destacando la cooperación interbancaria entre los bancos de desarrollo de los BRICS en moneda local y la cooperación interbancaria en relación a la agencia de rating (BRICS, 2017, \$10); d) se llegó a un acuerdo para crear una oficina del NBD en Sudáfrica, la primera oficina regional (BRICS, 2017, \$11); e) se estableció el sistema de intercambio de información macroeconómica del ARC (BRICS, 2017, §31).

La cumbre de los BRICS de Johannesburg, en Julio de 2018, tuvo como principal tema la $4^{\circ}$ Revolución Industrial o Revolución 4.0 (BRICS, 2018). Los objetivos primordiales están todos ligados a la expansion económica china: a) la profundización de la cooperación económica-comercial entre los países miembros para un crecimiento inclusivo en el contexto de los avances de la cuarta revolución industrial; b) la expansion institucional del NBD con la creación de una oficina en São Paulo, Brasil y; c) la consolidación de un BRICS plus, que significa, la posibilidad de expandir la formación económica y política de los BRICS en un formato aún no determinado 5 .

La expansion de los BRICS, partiendo de una iniciativa china, estuvo en la agenda de la cumbre de Xiamen. El Canciller Wang Yi llegó a sugerir la incoporación de México, Pakistan y Sri Lanka con el objetivo de crear una sociedad más amplia de países en desarrollo como una plataforma más activa de cooperacón Sur-Sur (CSS).

\section{China y el proceso de subsunción de los BRICS}

El surgimiento y evolución institucional de la formación económica y política BRICS, compuesta por cinco potencias emergentes y regionales, pre-

5 Podría ser en un formato de ampliación de miembros o, de otro modo, que permanezcan los 5 con otros invitados en la sucesivas cumbres. 
senta una interesante paradoja. En la medida en que la formación BRICS se consolida institucionalmente, con señales de ampliación, esta evolución fue sufriendo un proceso de subsunción ${ }^{6}$ institucional acoplada a una formación institucional mayor, producto de transfomaciones estructurales de alcance global liderada por la RPC. Se puede observar este processo en su amplitud siguiendo el esquema tripartito de la hegemonía de Robert Cox (1981) (Fig. 1). Este esquema ilustra el caso especial de la ascesión de China y el proceso de subsunción de la formación económica y política de los BRICS.

\section{Figura 1 Estructura histórica y dialéctica de la hegemonía de Robert Cox}

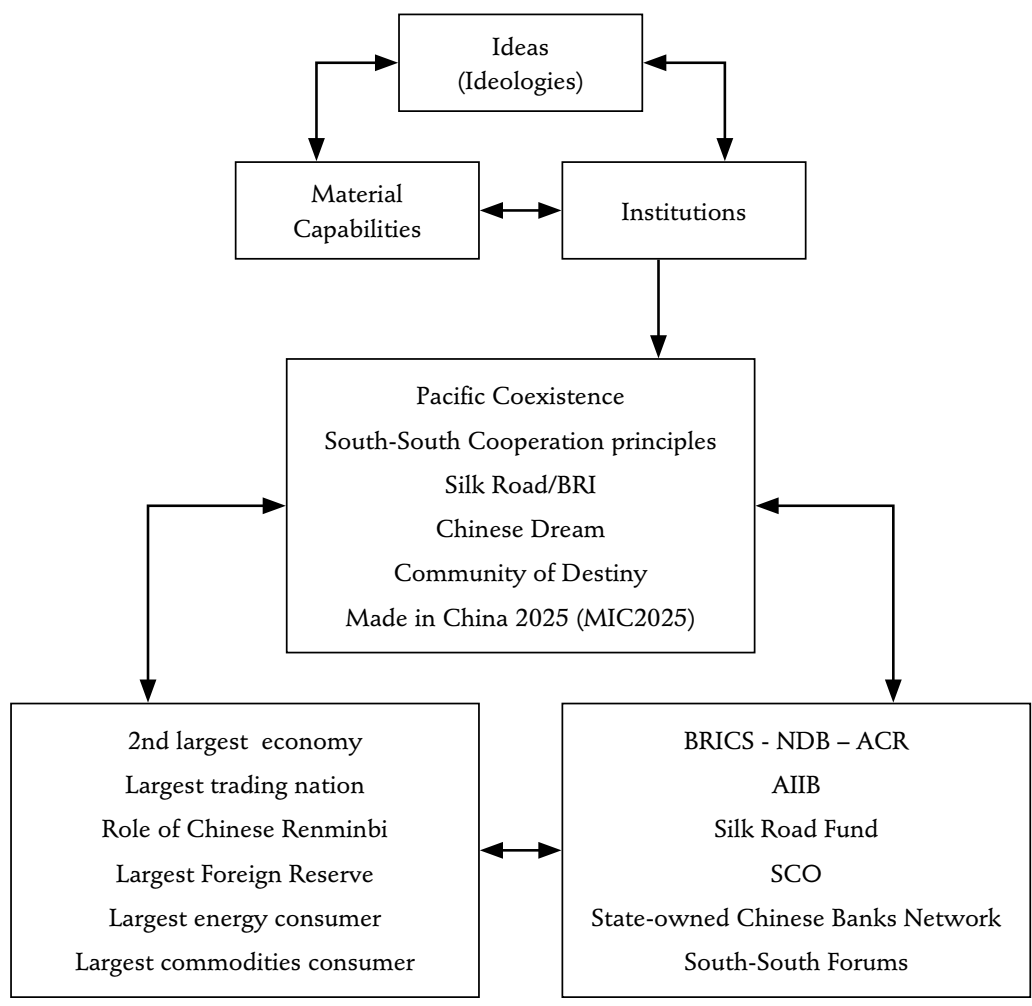

Source: Realizada por el autor y basada en la estructura dialéctica de la hegemonía de Robert Cox (1981) relacionada al proceso de expansión china basada en la figura de Li Xing (2019b). 
BRICS, como formación política-económica e institucional, constituye el amálgama y medio que perpetúa un orden mundial determinado en transformación. Este orden, como "configuración particular de fuerzas que sucesivamente definen la problemática de la guerra y la paz para el conjunto de los Estados" (Cox, 1981), es entendido a partir de sus conexiones estructurales del esquema dialéctico tripartito: las "ideas", es decir, las imágenes colectivas del orden social mundial'; las capacidades materiales; y las normas e instituciones específicas que la RPC fue desarrollando en el transcurso del siglo XXI. El rol de China fue destacado y analizado por Li Xing en su análisis sobre el BRI (Li, 2019b), no obstante, cabe a este trabajo enfocar esta paradoja en lo que respecta a la evolución de la formación BRICS.

La evolución institucional de los BRICS no presenta evidencias de un debilitamiento, que en una interpretación simple derivaría en inoperancia, disminución de la participación de los miembros o extinction institucional7 ${ }^{7}$ BRICS sigue su rumbo, a pesar que dos de sus miembros estén atravesando por crisis económicas y políticas (Brasil y Sudáfrica). Después de la inclusion de Sudáfrica, varios analistas excépticos, entre ellos el creador del acrónimo, Jim O'Neall, han destacado la inconsistencia de los BRICS (Naidoo, 2012; Vadell e Ramos, 2019). Otros analistas señalaron la incosistencia por el hecho de Rusia pertenecer al grupo (Cooper, 2006; Macfarla-

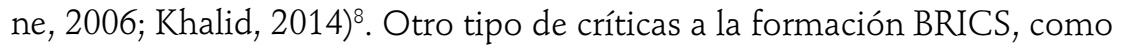
la de Kiely (2016), cuestionó el debilitamiento o declinación de los EEUU, presentando un escenario de escepticismo para los BRICS. Kiely afirma que "hay buenas razones para creer que esta supuesta emergencia estuvo basada en un discurso unilateral, y más aún, que ahora estamos entrando en un período en el que podemos hablar menos sobre la ascensión de las potencias emergentes y más sobre una crisis de mercados emergentes" (Kiely, 2016, p. 3).

Estas críticas registradas se muestran inconsistentes con los avances institucionales de la formación BRICS y con el proceso de ampliación (outreach) (Vadell e Ramos, 2019). Sin embargo, la paradoja es que la formación política y económica BRICS, en su proceso de consolidación institucional, fue gradualmente incorporándose a otro amalgama normativo-intitucional liderado por la RPC que estimuló la creación de otras instituciones que se

7 Como ejemplo de debilitamiento se puede destacar la evolución de la UNASUR reciente. 8 Russia is increasing its economic links with China, embodied in cooperation and energy and security agreements. (Ambrosio, 2017; Wu, 2017). 
complementan al NBD y al BRICS plus (fig. 1). Es decir: el BAII, el Shanghai Cooperation Organization (SCO), FRS, la red de Bancos Estatales de China - donde se destacan el China Development Bank (CDB) y el Export-Import Bank of China - y los importantes Foros de Cooperación Sur-Sur: Foro de Cooperación China-Africa (FOCAC) creado en el 2000; China-Caribbean Economic and Trade Cooperation Forum (CCETCF), creado en 2003; Forum for Economic and Trade Co-operation between China and Portuguese-Speaking Countries (FETCCPC), creado en 2003; China Arab-Sates Cooperation Forum (CASCF), creado en 2004; China-Pacific Island Countries Forum (CPICF), creado en 2006; y el Forum China-CELAC, creado oficialmente en la primera reunión ministerial en 2015.

La esfera ideacional está basada en los princípios de la CSS de la política externa china, que se remontan a la conferencia de Bandung en 1955 y al discurso de Zhou Enlai en Senegal en 1964 (Domínguez Martín, 2016; Hong, 2017; Domínguez Martín, 2018; Vadell, 2019). Los cinco princípios de coexistencia pacífica y los beneficios mútuos han sido la orientacion principal de la política externa China desde la Revolución de 1949. Aún en el siglo XXI, la RPC se presenta y se auto-identifica frente al mundo como una "Gran Nación en desarrollo", como lo combrueban los documentos oficiales (China, 2011; China-Scprc, 2011; 2014). El Gobierno de Xi Jinping le dio un nuevo empuje a la política externa, sin renunciar a los principios destacados. El Sueño Chino (Li e Shaw, 2014; Li, 2015) y el lanzamiento de la Nueva Ruta de la Seda, el BRI, le dieron un impulso inédito a la expansion de China.

El documento del gobierno chino "Vision and Actions" de 2015 destaca que la RPC no quiere restablecer la antigua Ruta de la Seda, sino utilizar la metáfora de: "el espíritu de la Ruta de la Seda", que remite a la antigüedad, como significado cultural para estimular la cooperación internacional (Liu e Dunford, 2016, p. 4). El Presidente chino, Xi Jinping, destacó que el BRI está inspirado en la antigua Ruta de la Seda, pero no pretende replicar ese espacio geográfico de comercio, sino ampliarlo para el mundo todo y abierto a todos los países:

(...)The Belt and Road Initiative is rooted in the ancient Silk Road. It focuses on the Asian, European and African continents, but is also open to all other countries. All countries, from either Asia, Europe, Africa or the Americas, can be international cooperation partners of the Belt and Road Initiative. The pursuit of this initiative is based on extensive consultation and its benefits will be shared by us all. (Xi, 2017) 
Si bien no podemos profundizar sobre la política externa official de China bajo el gobierno de Xi Jinping, es importante destacar su característica más asertiva, no obstante, sin renunciar a los principios heredados de Deng Xiaoping, basados en el postulado de mantener el bajo perfil para fomentar el desarrollo nacional (Oin, 2014).

\section{Consideraciones finales}

Las RRII y la EPI se deparan con un nuevo desafío para comprender las transformaciones económicas y el movimiento de placas téctonicas de la política internacional frente a la emergência de nuevos actores. Algunas expresiones intentan inspirarse en el pasado para entender el presente, por ejemplo: "nueva guerra fría". Otros recurren a la física y al concepto de "entropía" (Schweller, 2014). La idea de un mundo multipolar, generalmente viene acompañada de temores frente a las cuestiones culturales derivadas del rol económico cada vez más importante de países como China e India. Frente a estas transformaciones y a la inceridumbre que este proceso suscita, el fenómeno de los BRICS es sólo una manifestación, un síntoma importante de estos cambios.

El artículo pretendió interpretar los BRICS a partir del cuestionamiento que esta iniciativa representa para el orden liberal económico internacional y cómo este proceso puede afectar la inserción global de otros países. Nuestras conclusiones destacan una paradoja. Si bien no podemos afirmar que el grupo BRICS propone un nuevo paradigma de desarrollo, sin embargo, sí se observa una gradual y consistente consolidación institucional por intermedio del NBD y una gran posibilidad de expansion del grupo con un BRICS plus.

No obstante, esta institucionalización no está aislada de las transformaciones globales que tienen que ver con la ascensión de la RPC y su rol cada vez más importante dentro del grupo BRICS y en la economía política global. El NBD no puede disociarse del abordaje chino sobre cooperación SurSur en consonancia con el proceso de ascensión de China y el desarrollo de un entramado de red de poder financiera cada vez más interconectada con el proyecto BRI. El concepto de Acharya de "mundo multiplex" ayuda a reforzar la idea de este artículo, en la medida que detecta una especificidad del sistema internacional actual: "en el cual elementos del orden liberal 
sobreviven, pero son subsumidos en un complejo atravesado de múltiples órdenes internacionales transversales" (Acharya, 2017).

Esta metamorfosis de un orden liberal Occidental hacia un orden multiplex posee características específicas dialectamente enlazadas con el processo de ascensión de China y su propuesta ambiciosa de una especie de orden liberal incrustado (embedded liberal order) con características chinas, que este trabajo deja en abierto para investigaciones y proyectos futuros. Para finalizar, la figura 1, que detalla este proceso en el esquema de hegemonía de Cox, contempla las fuerzas materiales que dan sentido a la tríade dialéctica. China se transformó en la segunda mayor economía del mundo en 2010 y en tendencia creciente (Moore, 2011). En el año 2013, la RPC se torna la mayor nación comercial, superando a los EEUU (Monaghan, 2014). En el aspecto monetario, si bien la preminencia del dolar es una realidad y el Yuan está por detrás de la moneda estadounidense y del euro en los pagos internacionales, el creciente rol del Yuan es una tendencia fuerte, con pasos concretos para hacer de la moneda china un vehículo monetario de facilitación de operaciones comerciales por intermedio de acuerdos swaps con más de 30 países. En esa dirección de fortaleza creciente del la moneda china, en octubre de 2016, el FMI incluyó el Yuan en la cesta de monedas de los derechos especiales de giro. A esto le debemos acrecentar que, desde el 2008, China posee la mayor cantidad de reservas monetarias del mundo. Por añadidura, el gigante asiático es el mayor consumidor de energía y de commodities del mundo.

El BRICS continua su rumbo, no obstante, la agenda del grupo coincidirá con los imperativos de la expansion de China en el sistema capitalista contemporáneo. El estímulo al comercio intragrupo y cooperación económica más equilibrada podrá ser de beneficio mútuo, ya que actualmente el focal point comercial de los BRICS apunta a China. En el transcurso del siglo XXI, la RPC se fue consolidando como el principal socio comercial del resto de los miembros BRICS: Brasil, Rusia, Índia ${ }^{9}$ y Sudáfrica, con importantes superavits comerciales a favor China. Lo mismo sucede con el fortalecimiento institucional del NBD, que abrirá una oficina en Brasil. Por otro lado, el BRICS plus, en el formato que quede establecido, y la agenda de la Revolución Industrial 4.0 son una prioridad de la iniciativa BRI, el gran proyecto chino de una "Nueva Ruta de la Seda" de carácter global.

9 China es el primer sócio importador de India y el cuarto sócio exportador. En montos de dólares constituye el primer sócio comercial. 


\section{References}

ACHARYA, A. After Liberal Hegemony: The Advent of a Multiplex World Order. Ethics \& International Affairs, 31(3): 271-285, 2017.

ALEXANDROFF, A. S.; COOPER, A. F. Rising states, rising institutions: challenges for global governance. Waterloo, Ont./Washington, D.C.: Brookings Institution Press, 2010.

ALLISON, G. Destined for War: Can America and China Escape Thucydides's Trap? Boston/New York: Houghton Mifflin Harcourt 2017.

AMBROSIO, T. The Architecture of Alignment: The Russia-China Relationship and International Agreements. Europe-Asia Studies, 69(1): 110-156, 2017.

BIELER, A.; MORTON, A. D. Theoretical and Methodological Challenges of neo-Gramscian Perspectives in International Political Economy. International Gramsci Society Online Article 2003. Disponível em: http://www.internationalgramscisociety.org/resources/online_articles/main/main.html.

BIELSCHOWSKY, R. Cinqüenta anos de pensamento na CEPAL. São Paulo: Record, CEPAL, 2000.

BRI. Full text: Action plan on the Belt and Road Initiative. English Beijing. Mar 30 2015. Disponivel em: http://english.gov.cn/archive/publications/2015/03/30/content_281475080249035.htm.

BRICS. First Summit: Joint Statement of the BRIC Countries Leaders. Yekaterinburg: Ministério de Relações Exteriores. Jun 16 2009. Disponível em: http://brics.itamaraty.gov.br/pt_br/ categoria-portugues/20-documentos/73-primeiro-declaracao.

BRICS. Second Summit: Joint Statement. Brasilia: Iramaraty. April 16 2010. Disponível em: http://brics.itamaraty.gov.br/category-english/21-documents/66-second-summit.

BRICS. Third Summit: Sanya Declaration and Action Plan. Sanya: Itamaraty. April 14 2011. Disponível em: http://brics.itamaraty.gov.br/category-english/21-documents/67-third-summit.

BRICS. Fourth Summit: Delhi Declaration and Action Plan. New Delhi: Itanaraty. March 29 2012. Disponível em: http://brics.itamaraty.gov.br/category-english/21-documents/68-fourth-summit.

BRICS. Fifth Summit: Thekwini Declaration and Action Plan. Durban: Itamaraty. March 27 2013. Disponível em: http://brics.itamaraty.gov.br/category-english/21-documents/69-fifth-summit.

BRICS. Sixth Summit: Fortaleza Declaration and Action Plan. Fortaleza: Itamaraty. July 162014. Disponível em: http://brics.itamaraty.gov.br/category-english/21-documents/223-sixthsummit-declaration-and-action-plan.

BRICS. VII BRICS Summit - Ufa Declaration. Ufa: Itamaraty. July 9 2015. Disponível em: http://brics.itamaraty.gov.br/category-english/21-documents/253-vii-brics-summit-ufadeclaration.

BRICS. $8^{\text {th }}$ BRICS Summit Goa Declaration Goa: Itamaraty. Oct 16 2016. Disponivel em: http:// brics.itamaraty.gov.br/images/pdf/GoaDeclarationandActionPlan.pdf.

BRICS. BRICS Leaders Xiamen Declaration 2017. Disponível em: http://www.brics.utoronto. $\mathrm{ca} /$ docs/170904-xiamen.pdf.

BRICS. 10 th BRICS Summit Johannesburg Declaration. July 26 2018. Disponível em: https:// www.mea.gov.in/bilateral-documents.htm?dtl/30190/10th_BRICS_Summit_Johannesburg_Declaration. 
BROOKS, S. G.; IKENBERRY, J. G.; WOHLFORTH, W. C. Don't Come Home, America. The Case against Retrenchment. International Security, 37(3): 7-51, 2012/13.

CHIN, G. T. The State of the Art: Trends in the Study of the BRICS and Multilateral Organizations. In: LESAGE, D. e VAN DE GRAAF, T. (Ed.). Rising Powers and Multilateral Institutions. Basingstoke/New York: Palgrave Macmillan, 2015.

CHINA. China's Policy Paper on Latin America and the Caribbean 2008. Disponivel em: http:// www.gov.cn/english/official/2008-11/05/content_1140347.htm.

CHINA. White Paper on China's Peaceful Development. CHINA, S. C. O. T. P. S. R. O. Beijing 2011. Disponível em: http://www.china.org.cn/government/whitepaper/node_7126562.htm.

CHINA. China's second Africa policy paper: Xinhua 2015. Disponível em: http://news.xinhuanet.com/english/2015-12/04/c_134886545.htm.

CHINA-SCPRC. White Paper China's Foreign Aid. Beijing: State Council of the People's Republic of China 2011. Disponível em: http://english.gov.cn/archive/white_paper/2014/09/09/ content_281474986284620.htm.

CHINA-SCPRC. White Paper China's Foreign Aid: State Council of the People's Republic of China 2014. Disponível em: http://english.gov.cn/archive/white_paper/2014/08/23/content_281474982986592.htm.

CHRISTENSEN, S. F.; LI, X. The Emerging Powers and the Emerging World Order: Back to the Future? In: CHRISTENSEN, S. F.; LI, X. (Ed.), Emerging Powers, Emerging Markets, Emerging Societies. New York: Palgrave Macmillan, 2016.

COHEN, B. J. Advanced Introduction to International Political Economy. Northampton: Edward Elgar Publishing, 2014.

COOPER, A. F. Niche diplomacy: middle powers after the Cold War. Houndmills, Basingstoke, Hampshire/New York: Macmillan; St. Martin's Press, 1997.

COOPER, A. F. Squeezed or revitalised? Middle powers, the G20 and the evolution of global governance. Third World Quarterly 34(6): 963-984, 2013.

COOPER, A. F.; HIGGOTT, R. A. Middle power leadership and coalition building: the Cairns Group and the Uruguay Round, March 1990. Canberra: Research School of Pacific Studies, Australian National University, 1990.

COOPER, A. F.; HIGGOTT, R. A.; NOSSAL, K. R. Relocating middle powers: Australia and Canada in a changing world order. Vancouver: UBC Press, 1993.

COOPER, J. M. Russia as a BRIC: Only a dream? European Research Working Paper Series: Centre for Russian and East European Studies, University of Birmingham. No 13 2006. Disponível em: http://www.download.bham.ac.uk/govsoc/eri/working-papers/wp13-cooper.pdf.

COSTA VAZQUEZ, K. Can the BRICS propose a new development paradigm? Aljazeera. July 25 2018. Disponível em: https://www.aljazeera.com/indepth/opinion/brics-propose-development-paradigm-180718121646771.html.

COX, R. W. Social Forces, States and World Orders: Beyond International Relations Theory. Millennium - Journal of International Studies 10(2): 126-155, 1981.

COX, R. W Middlepowermanship, Japan, and Future World Order. International Journal 44, (4): 823-862, 1989. 
DOMÍNGUEZ MARTÍN, R. En los pliegues de la historia: Cooperación Sur-Sur y procesos de integración en América Latina y el Caribe Estudos Internacionais 4(2): 57-77, 2016.

DOMÍNGUEZ MARTÍN, R. China y el Renacimiento de África. In: VADELL, J. (Ed.). A expansão econômica e geopolítica da China no século XXI. Belo Horizonte: Editora PUC Minas, 2018.

GIACCAGLIA, C. Estrategias de "quodlíbet» en el escenario internacional contemporáneo: las acciones de India, Brasil y Sudáfrica (IBSA) en los ámbitos multilaterales. Revista Brasileira de Política Internacional 55(2): 90-108, 2013.

GILL, S. American hegemony and the Trilateral Commission. Cambridge/New York: Cambridge University Press, 1990.

GILLEY, B.; O'NEIL, A. Middle powers and the rise of China. Washington, DC: Georgetown University Press, 2014.

GILPIN, R. The political economy of international relations. Princeton, N.J.: Princeton University Press, 1987.

GILPIN, R. The challenge of global capitalism: the world economy in the $21^{\text {st }}$ century. Princeton, New Jersey: Princeton University Press, 2000.

GILPIN, R. Global political economy: understanding the international economic order. Princeton, N.J.: Princeton University Press, 2001.

HONG, Z. E. China's Foreign Aid: 60 years in retrospect. Singapore: Springer, 2017.

HUELSZ, C. Middle Power Theories and Emerging Powers in International Political Economy: A Case Study of Brazil. 2009. (PhD). Faculty of Humanities, PhD Thesis - University of Manchester - Faculty of Humanities, Manchester. Disponível em:

HUNTINGTON, S. P. Political order in changing societies. New Haven ; London: Yale University Press, 1968.

HURRELL, A. Paths to power: foreign policy strategies of intermediate States. Some reflections on the role of intermediate powers in international institutions. HURRELL, A.;COOPER, A. F., et al. Washington, D.C.: Latin American Program, Woodrow Wilson International Centre. 244: 23-41 p. 2000.

IKENBERRY, J. G. Why the Liberal World Order Will Survive. Ethics \& International Affairs 32(1): 17-29, 2018.

JESSOP, B.; OVERBEEK, H. Transnational Capital and Class Fractions. The Amsterdam School Perspective Reconsidered. New York: Routledge, 2019. 300.

JORDAAN, E. The emerging middle power concept: Time to say goodbye? South African Journal of International Affairs 24(3): 1-18, 2017.

KEOHANE, R. O. After hegemony: cooperation and discord in the world political economy. Princeton, N.J.: Princeton University Press, 1984.

KHALID, A. The power of the BRICS in world trade and growth, analysing the macroeconomic impacts within and across the bloc. In: LO, V. I. e HISCOCK, M. (Ed.). The rise of the BRICS in the global political economy: Changing paradigms? Cheltenham: Edward Elgar, 2014.

KIELY. The Rise and Fall of Emerging Powers. London: Palgrave Macmillan, 2016.

KIM, W.; GATES, S. Power transition theory and the rise of China. International Area Studies Review 18(3): 219-226, 2015. 
KINDLEBERGER, C. The world in depression, 1929-1939. Berkeley,: University of California Press, 1973.

KINDLEBERGER, C. Dominance and Leadership in International Economy. International Studies Quarterly 25(2): 242-254, June 1981.

KRAUTHAMMER, C. The Unipolar Moment. Foreign Affairs 1990. Disponível em:

LAYNE, C. The US-Chinese power shift and the end of the Pax Americana. International Affairs 94(1): 89-111, 2018.

LI, X. Interpreting and Understanding "The Chinese Dream" in a Holistic Nexus. Fudan J. Hum. Soc. Sci. 8(4):,505-520, 2015

LI, X. The Rise of Emerging Powers \& China and the Enlargement of 'Room for Maneuver' and 'Upward Mobility'. Rising Powers in Global Governance. 2017. Disponível em:

LI, X. Mapping China's 'One Belt One Road' Initiative. London/New York: Palgrave Macmillan, 2019a.

LI, X. Understanding the Multiple Facets of China's "One Belt One Road" Initiative. In: LI, X. (Ed.). Mapping China's 'One Belt One Road' Initiative. London/New York: Palgrave Macmillan, 2019b.

LI, X.; SHAW, T. M. "Same Bed, Different Dreams" and "Riding Tiger" Dilemmas: China's Rise and International Relations/Political Economy. Journal of Chinese Political Science 19(1): 69-93, 2014.

LIU, W.; DUNFORD, M. Inclusive globalization: unpacking China's Belt and Road Initiative. Area Development and Policy 1(3): 323-340, 2016.

MACFARLANE, N. S. The "R" in BRICs: Is Russia na emerging power? International Affairs 82(2): 41-57, 2006.

MEARSHEIMER, J. China's Unpeaceful Rise. Current History 105(690): 160-162, 2006.

MEARSHEIMER, J. The Gathering Storm: China's Challenge to US Power in Asia. Chinese Journal of International Politics, 3(4): 381-396, 2010.

MEARSHEIMER, J. The tragedy of great power politics. New York/London: W.W. Norton, 2001.

MONAGHAN, A. China surpasses US as world's largest trading nation. The Guardian. January 10, 2014. Disponível em: https://www.theguardian.com/business/2014/jan/10/chinasurpasses-us-world-largest-trading-nation.

MOORE, M. China is the world's second largest economy. The Telegraph. February 14, 2011. Disponível em: https://www.telegraph.co.uk/finance/economics/8322550/China-is-theworlds-second-largest-economy.html.

NAIDOO, S. South Africa's presence 'drags down Brics'. Mail \& Guardian 2012. Disponível em: https://mg.co.za/article/2012-03-23-sa-presence-drags-down-brics.

NAYYAR, D. BRICS, developing countries and global governance. Third World Quarterly 37(4): 575-591, 2016.

NOLTE, D. How to Compare Regional Powers: Analytical Concepts and Research Topics. ECPR Joint Session Workshops. Helsinki 2007. Disponível em: http://ecpr.eu/Filestore/ PaperProposal/212a550d-597b-4f60-86df-ec73a8e43707.pdf. 
ORGANSKI, A. F. K. World Politics. Second Edition. New York: Alfred A. Knopf, 1968.

QIN, Y. Continuity through Change: Background Knowledge and China's International Strategy. The Chinese Journal of International Politics 7(3):, 285-314, 2014.

RAMOS, L. et al. A Governança econômica global e os desafios do G-20 pós-crise financeira: análise das posições de Estados Unidos, China, Alemanha e Brasil. Revista Brasileira de Politica Internacional, 55(2): 10-27, 2012.

ROBERTS, C.; ARMIJO, L.; KATADA, S. The BRICS and Collective Financial Statecraft (Kindle edition). Oxford: Oxford University Press, 2018.

ROSTOW, W. W. The stages of economic growth, a non-Communist manifesto. Cambridge Eng.: University Press, 1960.

SCHOLTE, J. A. Globalization: a critical introduction. New York: St. Martin's Press, 2000.

SCHWELLER, R. The Age of Entropy, or Why the New World Order Won't be Orderly. Foreign Affairs. June 26, 2014.

STRANGE, S. International Economics and International Relations: A Case of Mutual Neglect. International Affairs 46(2): 304-315, 1970.

VADELL, J. China in Latin America: South-South Cooperation with Chinese Characteristics. Latin American Perspectives 4(2): 107-125, 2019.

VADELL, J.; RAMOS, L. The Role of Declining Brazil and Ascending China into the BRICS Initiative. In: LI, X. (Ed.). The International Political Economy of the BRICS. London: Routledge, 2019.

VIVARES, E. Regionalism, Development and the Post-Commodities Boom in South America. London: Palgrave Macmillan, 2018.

WALLERSTEIN, I. World-Systems Analysis. An Introduction. Durham and London: Duke University Press, 2004.

WANG, H. From "Taoguang Yanghui" to "Yousuo Zouwei": China's Engagement in Financial Minilateralism. CIGI Papers: CGI 2014. Disponível em: https://www.cigionline.org/sites/default/files/cigi_paper_no52.pdf.

WANG, H. China and International Financial Standards: From "Rule Taker" to "Rule Maker"? CIGI Papers. August 2018. Disponível em: https://www.cigionline.org/sites/default/files/documents/Paper\%20no.182_0.pdf.

WILLIAMSON, J. What Washington means by policy reform. In: WILLIAMSON, J. (Ed.). Latin American Adjustment: How Much Has Happened? Washington: Institute for International Economics, 1990.

WU, D. Russia-China Relations Reach a New High. The Diplomat: July 07 2017. Disponível em: https:/thediplomat.com/2017/07/russia-china-relations-reach-a-new-high/.

XI, J. Full text of President Xi's speech at opening of Belt and Road forum. Xinhuanet. May 142017. Disponível em: http://www.xinhuanet.com/english/2017-05/14/c_136282982.htm.

YAN, X. From Keeping a Low Profile to Striving for Achievement. The Chinese Journal of International Politics 7(2): 153-184, 2014. 


\section{Sobre el autor}

Javier Vadell - javier.vadell@gmail.com

Pontifícia Universidade Católica de Minas Gerais, Belo Horizonte, Minas Gerais, Brasil ORCID: https://orcid.org/0000-0002-5398-6083.

\section{Sobre el articulo}

Artículo recibido el 11 de abril de 2019. Aceptado para publicación el 12 de agosto de 2019. 